\title{
Strategies to prevent persistent or relapsed mixed cryoglobulinemia
}

Gonçalo Boleto, $\mathrm{MD}^{1,2, ;}$ David Saadoun, $\mathrm{PhD}^{1,2,3,4}$, Patrice Cacoub $\mathrm{MD}$, $\mathrm{PhD}^{1,2,3,4}$

${ }^{1}$ AP-HP, Groupe Hospitalier Pitié-Salpêtrière, Department of Internal Medicine and Clinical Immunology, F-75013, Paris, France;

${ }^{2}$ Centre de Référence des Maladies Auto-Immunes et Systémiques Rares, Centre de Référence des Maladies Auto-Inflammatoires et de l'Amylose, France

${ }^{3}$ Sorbonne Université, UPMC Univ Paris 06, UMR 7211, InflammationImmunopathology-Biotherapy Department (DHU i2B), F-75005, Paris, France; ${ }^{4}$ INSERM, UMR_S 959, F-75013, Paris, France; CNRS, FRE3632, F-75005, Paris, France;

This paper includes: abstract 206 words, 2827 words, 58 references, 2 figures

Correspondence should be addressed to: Prof. Patrice Cacoub, AP-HP, Hôpital PitiéSalpêtrière, Department of Internal Medicine and Clinical Immunology, 83 boulevard de l’hôpital. F-75013, Paris, France

Phone: + (33)(1) 421780 09. Fax: + (33)(1) 42178033.

E Mail: patrice.cacoub@aphp.fr

\section{Competing interests}

Patrice Cacoub has received consulting and lecturing fees from Abbvie, Astra Zeneca, Bristol-Myers Squibb, Gilead, Glaxo Smith Kline, Janssen, Merck Sharp Dohme, Roche, Servier and Vifor.

David Saadoun has received consulting and lecturing fees from from Medimmune, 
Abbvie, Bristol Meyer Squibb, Celgene, Sanofi, Roche, Servier, Gilead, AstraZeneca and Glaxo Smith Kline.

Gonçalo Boleto declares no competing interests. 


\section{Abstract \\ Introduction}

Mixed cryoglobulinemia (MC) are immune complexes that can deposit in small and medium size arteries and cause systemic vasculitis called cryoglobulinemic vasculitis (CryoVas). CryoVas shows different clinical manifestations i.e. purpura, arthralgia and/or arthritis, skin ulcers, peripheral neuropathy, nephritis, and may progress to more life threatening illness. Hepatitis C virus (HCV) infection is the more frequent condition to be assessed in patients with MC, followed by connective tissue diseases and B-cell non-Hodgkin's lymphoma. In HCV-related cases, the mainstay of CryoVas treatment is interferon free antiviral therapy. However, a significant proportion of patients who show HCV eradication will develop persistent CryoVas manifestations needing treatment intensification.

\section{Areas covered}

This review highlights key advances, recent clinical trial updates and ongoing studies on the management of persistent and refractory CryoVas. Therapeutic strategies and treatment agents to manage the disease are described. A literature review was performed by searching for available research studies published before January 2020 on the Medline (PubMed) database.

\section{Expert opinion}

Antiviral therapy with direct antiviral agents is the mainstay of treatment for patients with HCV-associated CryoVas. B-cell depleting strategies, mainly with rituximab, is the main therapeutic option in severe and refractory cases of infectious and non-infectious CryoVas. Ongoing trials are currently exploring other targeted biological treatments in this setting. 
Key words: cryoglobulinemia, vasculitis, therapy, chronic hepatitis C, rituximab

\section{Highlights box}

- Treatment of cryoglobulinemic vasculitis (CryoVas) may target either the viral trigger hepatitis C virus (HCV) or the downstream B-cell lymphoproliferation.

- Aggressive therapy with direct-acting antivirals should be systematically considered for HCV-CryoVas patients.

- $\quad$ Rituximab (RTX) is an effective and safe option for severe CryoVas patients.

- Management of rituximab-refractory CryoVas remains a major challenge.

- B-cell-targeted therapies other than RTX or low-dose interleukin-2 are the most promising strategies for the management of refractory CryoVas cases. 


\section{Introduction}

Cryoglobulinemia is defined as the persistent presence in the serum of circulating cryoglobulins which are immunoglobulin complexes that precipitate at cold temperature and redissolve at $37^{\circ} \mathrm{C}$. According to Brouet's classification [1] three subtypes of cryoglobulins can be defined: type I (isolated monoclonal component), and type II and III also called mixed cryoglobulins (MC) which are immune complexes composed by a polyclonal immunoglobulin acting as antigen and an immunoglobulin either monoclonal (type II) or polyclonal (type III). Small-to-medium size vessel vasculitis also called cryoglobulinemic vasculitis (CryoVas) is related to MC and can cause an array of clinical manifestations (purpura, arthralgia and/or arthritis, skin ulcers, peripheral neuropathy, nephritis) and can be a life-threatening condition. The fundamental mechanism contributing to MC is aberrant autoantibody production by B cells and low grade B cell proliferation. Whereas type I cryoglobulins are almost always linked to B cell lymphoproliferative disorders, type II and III mixed cryoglobulins are related to chronic hepatitis $\mathrm{C}$ virus (HCV) infection in the majority of cases. Of note, cryoglobulinemia is found in $40 \%$ to $60 \%$ of $\mathrm{HCV}$ infected patients, and $10-35 \%$ of them will develop CryoVas manifestations [2]. The production of cryoglobulinemia is correlated with a long lasting HCV infection, older age and the degree of liver fibrosis [3]. Other chronic infectious disorders caused by human immunodeficiency viruses (HIV) or hepatitis B virus (HBV) or systemic autoimmune disorders [in particular Sjögren’s syndrome (SS) and systemic lupus erythematosus (SLE)] can also be associated with MC and CryoVas. Five to $20 \%$ of patients with primary SS may present MC and CryoVas; such patients are at higher risk of B-cell lymphoma and have a relatively poor survival [3-5]. Treating patients suffering from CryoVas should be a priority. Even if symptoms are often initially mild, the disease may progress to life-threatening widespread vasculitis with 
glomerulonephritis, heart, gut or central nervous system involvement [6,7]. HCVassociated CryoVas is linked to significant mortality with a 5-year survival rate of $75 \%$ [8]. This was confirmed in a study showing a significantly lower survival rate in HCVCryoVas patients compared with the expected survival of age and sex-matched subjects in the general population [9]. Aside from its specific symptoms, CryoVas patients also have an increased risk of developing B-cell lymphomas with an incidence rate 35 times higher than in the general population. Despite the success with interferon-free directacting antivirals (DAAs) the management of refractory HCV-CryoVas remains a challenge. In patients presenting with severe or relapsing disease an induction phase of immunosuppression if often necessary. Targeting both the B-cell arm of autoimmunity and the viral trigger with B-cell-depleting induction therapy with rituximab and optimal anti-HCV treatment appears logical in this setting. In non-HCV CryoVas the treatment depends of the severity of the clinical picture and of the associated aetiology. However, in a small proportion of CryoVas patients, no underlying cause can be identified and therefore therapy directed at the underlying aetiology cannot be performed [10].

Here, we review recent studies investigating therapeutic strategies to manage persistent or relapsed MC and CryoVas.

\section{Methods}

A literature review was performed by searching for available research studies published before January 2020 on the Medline (PubMed) database. The following English keywords were used: mixed cryoglobulinemia, mixed cryoglobulins, cryoglobulinemic vasculitis, and also abatacept, antiviral, azathioprine, belimumab, biological, biological diseasemodifying antirheumatic drugs, corticoid, corticosteroid, cyclophosphamide, directacting antiviral, glucocorticoid, immunomodulatory, immunosuppressive, 
immunosuppressant, interleukine-2, methotrexate, methylprednisolone, mycophenolate, mofetil, ofatumumab, plasma exchange, prednisone, rituximab, tocilizumab, tofacitinib, tumor necrosis factor-alpha. These words were combined using the Boolean operators 'AND' or 'OR'.

No limits were applied. Controlled clinical trials, original articles, and case series were analysed . When there were no controlled clinical trials, open studies, case reports or low quality studies were analyzed. We screened reference lists of relevant articles to ensure that no relevant publications were overlooked.

\section{Treatment strategy}

\subsection{HCV-related cryoglobulinemic vasculitis}

In HCV infected patients, CryoVas treatment should be initiated without delay and always include antiviral treatment. Management of these patients must take into consideration a case-by-case stratification according to the severity of vasculitis [11-13]. Patients with mild-to-moderate vasculitis and non-life-threatening symptoms (i.e. arthralgia, asthenia, purpura, sensory neuropathy) may be treated with antiviral therapy alone. Sustained suppression of HCV replication is associated with a significantly higher rate of complete clinical remission in patients with HCV-CryoVas [14]. The recent advent of interferon-free direct-acting antivirals (DAAs), which have now the potential to induce sustained virological response rates greater than 95\%, has dramatically changed the management of chronic HCV infection and HCV-CryoVas patients [15,16]. An initial open-label study evaluating the effectiveness and safety of an oral interferon-free and ribavirin-free regimen with sofosbuvir plus daclatasvir in patients with HCV-CryoVas showed a complete clinical remission in $90.2 \%$ of patients at week 24 . Complete remission rate at 12 months was $90 \%$, with an event free survival rate at 2 years of $100 \%$ 
(although the disappearance of cryoglobulin was noted in only 50\% of cases) [17]. Gragnani et al have also showed very high rates of clinical and virological responses (93\% and $100 \%$, respectively) in patients treated by sofosbuvir based interferon-free regimens [18]. Bonacci et al reported $71 \%$ of complete clinical response, $94 \%$ of sustained virological response and $48 \%$ of cryoglobulin clearance in a series of $35 \mathrm{HCV}$-CryoVas patients treated by different DAAs [19]. The most recently approved DAAs show improved pan-genomic and higher antiviral activities requiring no particular therapeutic guidance (i.e. duration of antiviral therapy, presentation and severity of CryoVas, HCV genotype, viral load and severity of fibrosis stage/hepatic function) [20].

Immunosuppressant therapy remains useful in patients with severe HCV-CryoVas in order to rapidly control vasculitis [11,12,21]. Of note, even in such severe CryoVas cases, DAAs should be always used to clear HCV infection. Intravenous methylprednisolone (0.5-1.0g/day for 3-5 days) in combination with plasma exchange (PLEX) should be used in life-threatening presentations such as severe kidney dysfunction, skin necrosis, involvement of the gastrointestinal tract or central nervous system [2]. The use of B-cell depleting agents such as rituximab has demonstrated greater efficacy than conventional immunosuppressants in HCV-CryoVas, even in cirrhotic patients [22]. The best rituximab dosing regimen is $375 \mathrm{mg} / \mathrm{m}^{2}$ given weekly for 4 consecutive weeks [23]. In a randomized controlled trial the authors compared rituximab versus conventional treatment (glucocorticoids; azathioprine or cyclophosphamide; or PLEX) in patients with severe HCV-CryoVas. Relapse-free survival at 3, 6 and 24 months was statistically higher in the rituximab group. Vasculitis activity as measured by the Birmingham Activity Score (BVAS) decreased only after treatment with rituximab. Interestingly, the median duration of response to rituximab was 18 months [22]. Rituximab provides long-term remission and besides its immunomodulatory effect, it also plays an important role by depleting 
CD19 positive-B cells, known to be HCV reservoirs [24].

Some HCV-CryoVas patients successfully treated with anti-viral therapy and undetectable viral load may present with persistent CryoVas manifestations. This effect is likely due to the emergence of clonal B-cells that developed a neoplastic behaviour after prolonged HCV stimulation, acquiring an independent proliferative potential. These patients should be treated with rituximab alone [2]. Caution is nonetheless needed regarding the possibility of rituximab associated vasculitis flare due to the formation of immune complexes [21]. A recent study reporting rituximab-associated autoimmune disease flares identified $3.4 \%$ vasculitis flares after a median of 8 days following rituximab infusion. All cases were observed in patients with type II MC after a median of 8 days following rituximab infusion. Vasculitis flares included acute kidney injury, purpura, gastrointestinal involvement and myocarditis. Among HCV-CryoVas patients, those who develop rituximab-associated CryoVas had more frequently renal involvement, B-cell lymphoproliferation, higher levels of cryoglobulin and lower C4 serum levels [25]. A possible way to overcome the risk of CryoVas flare is to use rituximab at lower dosage, as it seems to be as effective as higher dosages [26,27]. Interestingly, Saadoun et al showed that treatment with low-dose interleukin-2 lead to clinical improvement of HCVCryoVas through regulatory $\mathrm{T}$ cells recovery, which might also represent a future approach to manage refractory cases [28].

\subsection{Cryoglobulinemic vasculitis unrelated to HCV infection}

\subsubsection{Other infectious disorders}

Non-HCV related CryoVas secondary to other infectious disorders are rare. They are mainly represented by HIV and chronic HBV infection. MC can be detected in $17 \%$ and 9\% of patients with HIV and HBV respectively [9,29]. Only anecdotal cases of other 
infectious causes such cytomegalovirus, Epstein Barr virus, parvovirus B19, pyogenic bacterial and parasitic infections, have been reported [30-32]. In patients with CryoVas due to HBV or HIV, antiviral therapy should always be initiated before or at the same time as immunosuppressive therapy. Such patients are at higher risk for enhanced viral replication resulting in accelerated liver injury and worsening of extra-hepatic manifestations when treated with cyclophosphamide or rituximab [33]. Rituximab should not be used in patients with active hepatitis flares, as fatal hepatitis cases have been reported [34]. In patients with mild to moderate HBV-related CryoVas, antiviral agents (entecavir, tenofovir, alpha-interferon, lamivudine) should be used as first-line therapy. The therapeutic goal in these patients is to obtain a negativation of serum HBV-DNA [32]. In most severe vasculitic presentations, concomitant therapy with specific antivirals and rituximab and/or PLEX and/or conventional immunosuppressive agents (i.e. cyclophosphamide), in association with short-term glucocorticoids is recommended [35].

\subsubsection{Autoimmune disorders}

The widespread use of DAAs to treat HCV infection and HCV-CryoVas has deeply modified the spectrum of aetiologies causing CryoVas. From a large database of 15970 patients screened for the presence of cryoglobulinemia between 2011 and 2018, it has been showed that the incidence of MC is decreasing steadily. HCV infection represents in recent years only $56 \%$ of cases, and as a consequence, connective tissue diseases in particular SS and SLE represent the main aetiologies of MC [36]. This is in line with another recent study of 242 patients with non-infectious CryoVas, showing underlying connective tissue disorders (SS, SLE and systemic sclerosis) in 30\% of cases [37]. Two previous studies $[38,39]$ showed that MC were detected in the sera of $16 \%$ and $25 \%$ of patients with SS and SLE respectively. Moreover, patients with circulating cryoglobulins 
had more frequently CryoVas in particular cutaneous vasculitis. The presence of systemic vasculitis with cutaneous and peripheral nervous system involvement in patients with SS should prompt an evaluation for the presence of lymphoma. In the French database of 15 970 patients, SLE represented the main cause of non-related MC followed by SS; however, SS appeared as the first cause of CryoVas. In 2018, autoimmune disorders represented the main cause of MC (36.5\% of all cases) as compared to HCV infection (32.6\%) [36]. The approach for inducing clinical remission in these patients are derived from therapeutic strategies used in other systemic vasculitides. The therapeutic approach is mostly based on the use of high-dose glucocorticoids, and conventional immunosuppressive treatment such as cyclophosphamide and/or rituximab. Other agents such as azathioprine and mycophenolate mofetil are mainly used as remission maintenance agents. According to recent data, the use of glucocorticoids and rituximab may now be considered standard of care in patients with severe CryoVas secondary to autoimmune disorders, in particular connective tissue diseases [40,41].

\subsubsection{Haematological disorders}

In patients with non-infectious CryoVas, B-cell malignancy accounts for $\sim 20 \%$ of cases [37]. The most common haematological conditions associated with MC are B-cell nonHodgkin lymphomas, mainly marginal zone and lymphoplasmacytic lymphomas. The overall risk of non-Hodgkin lymphoma in patients with HCV-CryoVas is 35 times higher than in the general population [42]. HCV-positive non-Hodgkin's lymphoma usually occurs following a long period of infection ( $>15$ years) especially in the presence of cryoglobulin levels above $600 \mathrm{mg} / \mathrm{L}$, the presence of CryoVas manifestations and hypogammaglobulinemia [43]. Treating the underlying lymphoproliferative disorder should be a priority in the management of symptomatic CryoVas. The treatment usually 
consists of anthracycline-based chemotherapy coupled with rituximab (375 mg/m² given weekly for 4 consecutive weeks) [44]. Plasma exchange therapy may deserve consideration in patients with severe vasculitic symptoms [45].

\subsubsection{Essential}

In a small percentage of non-HCV-related MC, no underlying cause can be identified and patients are classified as having essential cryoglobulinemia [37]. Since therapy directed at the underlying aetiology cannot be performed, these patients should be treated with immunosuppressants which usually includes a short course of glucocorticoids associated with rituximab (less frequently cyclophosphamide or mycophenolate mofetil) [46,47]. PLEX can be considered in the most severe or refractory cases. Although rituximab has proven to be safer than conventional immunosuppressants and highly effective in this setting, some patients show no or only partial response to this treatment. Rituximabrefractory CryoVas can be defined as an insufficient response or no response within 4-6 weeks after the initial infusion. Before treatment is intensified, the clinician should always rule out CryoVas mimics (underlying infection or cancer-related comorbidity) and evaluate patient's adherence to medical prescriptions [2]. Some alternative approaches have been proposed in refractory cases. Ofatumumab (a potent anti-CD20 monoclonal antibody) or belimumab (an anti-B-cell activator antibody), are potential second-line therapies for patients who continue to show symptoms of CryoVas [48]. While tumor necrosis factor inhibitors are not effective, other biologic disease-modifying antirheumatic drugs such as abatacept or tocilizumab seem to be promising $[49,50]$.

\section{Expert opinion}

The introduction of safe and highly effective HCV therapies has resulted in a paradigm shift in how we manage refractory and relapsed HCV-CryoVas. Despite the excellent 
efficacy of DAAs, $3 \%$ to $10 \%$ of patients who achieved viral eradication can still experience CryoVas relapses $[17,18,51]$. Available data suggest that when the immune disorder is established, antivirals may not be able to interfere with the immune-mediated injury and the pathogenesis of CryoVas. The degree of reversibility of CryoVas is inversely correlated with the duration of the immune stimulation by HCV [52]. Lowgrade B-cell lymphoproliferation (i.e. persistence of B-cell clones) after HCV eradication probably explains persistent immunological abnormalities and vasculitic symptoms in some individuals with long lasting infection. A cohort study found that the effect of sustained virological responses against extrahepatic manifestations was lessened as the time to initiation of HCV therapy increased [53]. In some patients, B-cell stimulation and proliferation might have passed a "no return" point. This emphasizes the importance of the early treatment of HCV infection and CryoVas. Clinicians should be aware of the possibility of the presence of underlying malignant lymphoma when patients develop a relapse of CryoVas despite sustained virological responses [54]. An early use of DAAs is therefore recommended in all HCV-CryoVas patients. DAAs should be used alone to treat non-severe cases of CryoVas to eradicate the immune-mediated injury and prevent B-cell lymphoproliferation. In more severe cases, especially in the presence of kidney involvement, DAAs should be associated to immunosuppressants with rituximab being the most widely used drug. Rituximab also plays an important role by depleting CD19 positive-B-cells which are known to be HCV reservoirs [55]. According to several studies, rituximab is a safe medication in patients with CryoVas, including those with chronic HCV infection [48]. However, clinicians should be aware that some patients might present CryoVas flares after rituximab therapy especially in the presence of renal involvement, high levels of circulating cryoglobulin and low C4 serum levels. Indeed, rituximab may form complexes with IgM and IgG1 leading to vasculitis flare [25]. In 
patients with risk factors of rituximab-induced vasculitis flare, lower doses of rituximab should be used (250-500mg per infusion). We also recommend that all patients with CryoVas who are receiving immunosuppressive therapy should receive age-appropriate vaccination.

The management of rituximab-refractory CryoVas remains a major challenge in HCV and non-HCV patients. Refractory disease can be defined as the absence of CryoVas clinical response within 4-6 weeks after induction therapy, or less than 50\% improvement after 12 weeks [2]. In the most severe and refractory cases, we recommend the use of plasma exchange daily for 5 to 14 sessions, followed by 3 PLEX per week for two to three weeks. Of note, replacement fluid should be warmed to prevent precipitation of circulating cryoglobulin.

The management of severe CryoVas can be especially challenging in immunocompromised patients. As stated before, a fair proportion of patients with HIV or B-cell lymphoproliferative disorders present MC. A reduction in the serum levels of polyclonal immunoglobulins (Ig) is frequent in the latter increasing the risk of infectious complications [56]. Furthermore, secondary hypogammaglobulinemia is also a wellknown late complication after rituximab treatment [57]. Interestingly, a previous study showed that a specific clonal population of B cells resembling the exhausted CD21(low) B cells seen in HIV or in primary immunodeficiency conditions can accumulate in HCVassociated CryoVas [58]. In the context of immunodeficiency, induction of regulatory $\mathrm{T}$ cells with low-dose IL-2 for the treatment of refractory CryoVas represents a promising strategy [28].

Due to the rarity of this disorder, it is worthwhile mentioning that the majority of the studies for the management of relapsing CryoVas discussed in this review come from low level evidence uncontrolled observational or case series studies. 
Based on these data we propose an algorithm that can aid clinical decision making and pharmacological management of CryoVas (Figure 1). Figure 2 illustrates the pathophysiology and potential therapeutic targets of refractory/relapsing CryoVas.

\section{References}

Papers of special note have been highlighted as either of interest $\left(^{*}\right)$ or of considerable interest $(* *)$ to readers. 
[1] Brouet J-C, Clauvel J-P, Danon F, et al. Biologic and clinical significance of cryoglobulins: A report of 86 cases. Am. J. Med. 1974;57:775-788.

[2] Roccatello D, Saadoun D, Ramos-Casals M, et al. Cryoglobulinaemia. Nat. Rev. Dis. Primer. 2018;4:11.

[3] Zignego AL, Pawlotsky J-M, Bondin M, et al. Expert opinion on managing chronic HCV in patients with mixed cryoglobulinaemia vasculitis. Antivir. Ther. 2018;23:1-9.

[4] Tzioufas AG, Boumba DS, Skopouli FN, et al. Mixed monoclonal cryoglobulinemia and monoclonal rheumatoid factor cross-reactive idiotypes as predictive factors for the development of lymphoma in primary Sjögren's syndrome. Arthritis Rheum. 1996;39:767-772.

[5] Quartuccio L, Isola M, Baldini C, et al. Biomarkers of lymphoma in Sjögren's syndrome and evaluation of the lymphoma risk in prelymphomatous conditions: Results of a multicenter study. J. Autoimmun. 2014;51:75-80.

[6] Terrier B, Cacoub P. Cryoglobulinemia vasculitis: an update. Curr. Opin. Rheumatol. 2013;25:10-18.

[7] Cacoub P, Comarmond C, Domont F, et al. Cryoglobulinemia Vasculitis. Am. J. Med. 2015;128:950-955.

[8] Terrier B, Semoun O, Saadoun D, et al. Prognostic factors in patients with hepatitis C virus infection and systemic vasculitis. Arthritis Rheum. 2011;63:1748-1757.

[9] Ferri C, Sebastiani M, Giuggioli D, et al. Mixed cryoglobulinemia: demographic, clinical, and serologic features and survival in 231 patients. Semin. Arthritis Rheum. 2004;33:355-374.

[10] Saadoun D, Cacoub P. Current and future therapeutic strategies for treating mixed cryoglobulinemia. Expert Opin. Orphan Drugs. 2014;2:381-390.

[11] Retamozo S, Brito-Zerón P, Quartuccio L, et al. Introducing treat-to-target strategies of autoimmune extrahepatic manifestations of chronic hepatitis $C$ virus infection. Expert Rev. Clin. Pharmacol. 2017;10:1085-1101.

[12] Ramos-Casals M, Zignego AL, Ferri C, et al. Evidence-based recommendations on the management of extrahepatic manifestations of chronic hepatitis $\mathrm{C}$ virus infection. J. Hepatol. 2017;66:1282-1299.

[13] European Association for the Study of the Liver. Electronic address: easloffice@easloffice.eu. EASL Recommendations on Treatment of Hepatitis C 2016. J. Hepatol. 2017;66:153-194.

[14] Cacoub P, Desbois AC, Comarmond C, et al. Impact of sustained virological response on the extrahepatic manifestations of chronic hepatitis C: a meta-analysis. Gut. 2018;67:2025-2034.

[15] Cacoub P, Si Ahmed SN, Ferfar Y, et al. Long-term Efficacy of Interferon-Free 
Antiviral Treatment Regimens in Patients With Hepatitis C Virus-Associated Cryoglobulinemia Vasculitis. Clin. Gastroenterol. Hepatol. Off. Clin. Pract. J. Am. Gastroenterol. Assoc. 2019;17:518-526.

** Important study showing long-term efficacy of direct-acting antivirals in patients with hepatitis C virus (HCV)-associated cryoglobulinemic vasculitis (CryoVas).

[16] Landau D-A, Scerra S, Sene D, et al. Causes and predictive factors of mortality in a cohort of patients with hepatitis $\mathrm{C}$ virus-related cryoglobulinemic vasculitis treated with antiviral therapy. J. Rheumatol. 2010;37:615-621.

[17] Saadoun D, Pol S, Ferfar Y, et al. Efficacy and Safety of Sofosbuvir Plus Daclatasvir for Treatment of HCV-Associated Cryoglobulinemia Vasculitis. Gastroenterology. 2017;153:49-52.e5.

* Open-label, prospective, multicenter study of the effectiveness and tolerance of an oral interferon and ribaviran-free regimen in patients with HCV-CryoVas.

[18] Gragnani L, Visentini M, Fognani E, et al. Prospective study of guideline-tailored therapy with direct-acting antivirals for hepatitis C virus-associated mixed cryoglobulinemia. Hepatol. Baltim. Md. 2016;64:1473-1482.

[19] Bonacci M, Lens S, Londoño M-C, et al. Virologic, Clinical, and Immune Response Outcomes of Patients With Hepatitis C Virus-Associated Cryoglobulinemia Treated With Direct-Acting Antivirals. Clin. Gastroenterol. Hepatol. 2017;15:575-583.e1.

[20] Desbois AC, Cacoub P, Saadoun D. Cryoglobulinemia: An update in 2019. Joint Bone Spine. 2019;86:707-713.

[21] Ramos-Casals M, Stone JH, Cid MC, et al. The cryoglobulinaemias. The Lancet. 2012;379:348-360.

[22] Quartuccio L, Zuliani F, Corazza L, et al. Retreatment regimen of rituximab monotherapy given at the relapse of severe HCV-related cryoglobulinemic vasculitis: Long-term follow up data of a randomized controlled multicentre study. J. Autoimmun. 2015;63:88-93.

[23] Sneller MC, Hu Z, Langford CA. A randomized controlled trial of rituximab following failure of antiviral therapy for hepatitis $C$ virus-associated cryoglobulinemic vasculitis. Arthritis Rheum. 2012;64:835-842.

** Randomized study showing the superiority of rituximab compared to other immunosuppressants in CryoVas.

[24] Morsica G, Tambussi G, Sitia G, et al. Replication of Hepatitis C Virus in B Lymphocytes (CD19+). Blood. 1999;94:1138-1139.

[25] Desbois AC, Biard L, Sene D, et al. Rituximab associated vasculitis flare: incidence, predictors and outcome. J. Rheumatol. 2019;

* Retrospective study reporting the incidence, predictors, and outcome of rituximab- 
associated CryoVas flares.

[26] Visentini M, Granata M, Veneziano ML, et al. Efficacy of low-dose rituximab for mixed cryoglobulinemia. Clin. Immunol. Orlando Fla. 2007;125:30-33.

[27] Visentini M, Tinelli C, Colantuono S, et al. Efficacy of low-dose rituximab for the treatment of mixed cryoglobulinemia vasculitis: Phase II clinical trial and systematic review. Autoimmun. Rev. 2015;14:889-896.

[28] Saadoun D, Rosenzwajg M, Joly F, et al. Regulatory T-cell responses to low-dose interleukin-2 in HCV-induced vasculitis. N. Engl. J. Med. 2011;365:2067-2077.

** First pilot study showing the efficacy of an immunotherapy (low-dose interleukin-2) in HCV-CryoVas.

[29] Bonnet F, Pineau J-J, Taupin J-L, et al. Prevalence of cryoglobulinemia and serological markers of autoimmunity in human immunodeficiency virus infected individuals: a cross-sectional study of 97 patients. J. Rheumatol. 2003;30:20052010.

[30] Terrier B, Marie I, Lacraz A, et al. Non HCV-related infectious cryoglobulinemia vasculitis: Results from the French nationwide CryoVas survey and systematic review of the literature. J. Autoimmun. 2015;65:74-81.

[31] Galli M, Oreni L, Saccardo F, et al. HCV-unrelated cryoglobulinaemic vasculitis: the results of a prospective observational study by the Italian Group for the Study of Cryoglobulinaemias (GISC). Clin. Exp. Rheumatol. 2017;35 Suppl 103:67-76.

[32] Mazzaro C, Maso LD, Urraro T, et al. Hepatitis B virus related cryoglobulinemic vasculitis: A multicentre open label study from the Gruppo Italiano di Studio delle Crioglobulinemie - GISC. Dig. Liver Dis. 2016;48:780-784.

[33] Viganò M, Martin P, Cappelletti M, et al. HBV-Associated Cryoglobulinemic Vasculitis: Remission after Antiviral Therapy with Entecavir. Kidney Blood Press. Res. 2014;39:65-73.

[34] Li X, Lin Q, Dong M, et al. Prognostic analysis of acute exacerbations of hepatitisB after chemotherapy in combination with rituximab in 19 patients with lymphoma. Leuk. Lymphoma. 2010;51:1678-1685.

[35] Evens AM, Jovanovic BD, Su Y-C, et al. Rituximab-associated hepatitis B virus (HBV) reactivation in lymphoproliferative diseases: meta-analysis and examination of FDA safety reports. Ann. Oncol. Off. J. Eur. Soc. Med. Oncol. 2011;22:1170-1180.

[36] Boleto G, Ghillani-Dalbin P, Musset L, Cacoub P, Saadoun D. Mixed Cryoglobulinaemia Since the Advent of New Direct-acting Antivirals for Hepatitis C Infection: Clinical Characteristics, Etiologies and Biological Features in 679 Patients. [cited 2020 Jan 23]. Available from: Arthritis Rheumatol. 2019; 71 (suppl 10).

[37] Terrier B, Krastinova E, Marie I, et al. Management of noninfectious mixed 
cryoglobulinemia vasculitis: data from 242 cases included in the CryoVas survey. Blood. 2012;119:5996-6004.

* Large observational study on the treatment of non-HCV CryoVas.

[38] Ramos-Casals M, Cervera R, Yagüe J, et al. Cryoglobulinemia in primary Sjögren's syndrome: prevalence and clinical characteristics in a series of 115 patients. Semin. Arthritis Rheum. 1998;28:200-205.

[39] García-Carrasco M, Ramos-Casals M, Cervera R, et al. Cryoglobulinemia in systemic lupus erythematosus: prevalence and clinical characteristics in a series of 122 patients. Semin. Arthritis Rheum. 2001;30:366-373.

[40] Terrier B, Launay D, Kaplanski G, et al. Safety and efficacy of rituximab in nonviral cryoglobulinemia vasculitis: data from the French Autoimmunity and Rituximab registry. Arthritis Care Res. 2010;62:1787-1795.

[41] Terrier B, Marie I, Launay D, et al. Predictors of early relapse in patients with noninfectious mixed cryoglobulinemia vasculitis: results from the French nationwide CryoVas survey. Autoimmun. Rev. 2014;13:630-634.

[42] Monti G, Pioltelli P, Saccardo F, et al. Incidence and characteristics of nonHodgkin lymphomas in a multicenter case file of patients with hepatitis $\mathrm{C}$ virusrelated symptomatic mixed cryoglobulinemias. Arch. Intern. Med. 2005;165:101105.

[43] Saadoun D, Landau DA, Calabrese LH, et al. Hepatitis C-associated mixed cryoglobulinaemia: a crossroad between autoimmunity and lymphoproliferation. Rheumatology. 2007;46:1234-1242.

[44] Solimando AG, Ribatti D, Vacca A, et al. Targeting B-cell non Hodgkin lymphoma: New and old tricks. Leuk. Res. 2016;42:93-104.

[45] Vallisa D, Bernuzzi P, Arcaini L, et al. Role of Anti-Hepatitis C Virus (HCV) Treatment in HCV-Related, Low-Grade, B-Cell, Non-Hodgkin's Lymphoma: A Multicenter Italian Experience. J. Clin. Oncol. 2005;23:468-473.

[46] Roccatello D, Baldovino S, Rossi D, et al. Long-term effects of anti-CD20 monoclonal antibody treatment of cryoglobulinaemic glomerulonephritis. Nephrol. Dial. Transplant. Off. Publ. Eur. Dial. Transpl. Assoc. - Eur. Ren. Assoc. 2004;19:3054-3061.

* Long-term effects of rituximab in patients with HCV-CryoVas glomerulonephritis and severe sytemic vasculitis.

[47] Roccatello D, Sciascia S, Baldovino S, et al. Improved (4 Plus 2) Rituximab Protocol for Severe Cases of Mixed Cryoglobulinemia: A 6-Year Observational Study. Am. J. Nephrol. 2016;43:251-260.

[48] Ostojic P, Jeremic IR. Managing refractory cryoglobulinemic vasculitis: challenges and solutions. J. Inflamm. Res. 2017;10:49-54. 
[49] Joseph AM. Treatment of rheumatoid arthritis in patients with concomitant chronic hepatitis C infection. Ther. Adv. Musculoskelet. Dis. 2012;4:35-40.

[50] Cohen C, Mekinian A, Saidenberg-Kermanac'h N, et al. Efficacy of tocilizumab in rituximab-refractory cryoglobulinemia vasculitis. Ann. Rheum. Dis. 2012;71:628-629.

[51] Gragnani L, Fognani E, Piluso A, et al. Long-term effect of HCV eradication in patients with mixed cryoglobulinemia: a prospective, controlled, open-label, cohort study. Hepatol. Baltim. Md. 2015;61:1145-1153.

** Large prospective cohort study evaluating the long-term effects of viral eradication on HCV-mixed cryoglobulins.

[52] Zignego AL, Gragnani L, Visentini M, et al. Reply. Hepatol. Baltim. Md. 2017;65:1771-1772.

[53] Zignego AL, Pawlotsky J-M, Bondin M, et al. Expert opinion on managing chronic HCV in patients with mixed cryoglobulinaemia vasculitis. Antivir. Ther. 2018;23:1-9.

[54] Landau D-A, Saadoun D, Halfon P, et al. Relapse of hepatitis C virus-associated mixed cryoglobulinemia vasculitis in patients with sustained viral response. Arthritis Rheum. 2008;58:604-611.

[55] Dammacco F, Racanelli V, Russi S, et al. The expanding spectrum of HCV-related cryoglobulinemic vasculitis: a narrative review. Clin. Exp. Med. 2016;16:233-242.

[56] Vacca A, Melaccio A, Sportelli A, et al. Subcutaneous immunoglobulins in patients with multiple myeloma and secondary hypogammaglobulinemia: a randomized trial. Clin. Immunol. 2018;191:110-115.

[57] Boleto G, Avouac J, Wipff J, et al. Predictors of hypogammaglobulinemia during rituximab maintenance therapy in rheumatoid arthritis: A 12-year longitudinal multi-center study. Semin. Arthritis Rheum. 2018;48:149-154.

[58] Visentini M, Cagliuso M, Conti V, et al. Clonal B cells of HCV-associated mixed cryoglobulinemia patients contain exhausted marginal zone-like and CD21 low cells overexpressing Stra13. Eur. J. Immunol. 2012;42:1468-1476. 


\section{Legends}

Figure 1. Proposed algorithm for pharmacological treatment of cryoglobulinemia vasculitis (CryoVas). IV, intravenous; PLEX, plasma exchange; GC, glucocorticoids; IL2, interleukin-2. ${ }^{1}$ Severe kidney dysfunction, skin necrosis, involvement of the gastrointestinal tract or central nervous system; ${ }^{2}$ In cases of hepatitis B or HIV infection antiviral therapy should always be initiated before or at the same time as immunosuppressive therapy; ${ }^{3}$ In the presence of risk factors for rituximab-induced vasculitis flare (i.e. renal involvement, high levels of circulating cryoglobulin and low C4 levels) lower doses of rituximab should be used (250-500mg per infusion).

Figure 2. The main pathophysiology and potential therapy targets of cryoglobulinemic vasculitis. HCV, hepatitis C virus; Th cell, T-helper cell; BlyS/BAFF, B Lymphocyte Stimulator/ B-cell activating factor; IL-6, interleukin-6; IL-2, interleukin-2; Treg, regulatory $\mathrm{T}$ cells. 
Start IV methylprednisolone 0.5-1g/day for 3-5 days and PLEX daily for 5-14 sessions and then 3 sessions/week for 2-3 weeks ${ }^{2}$

\section{Phase II}
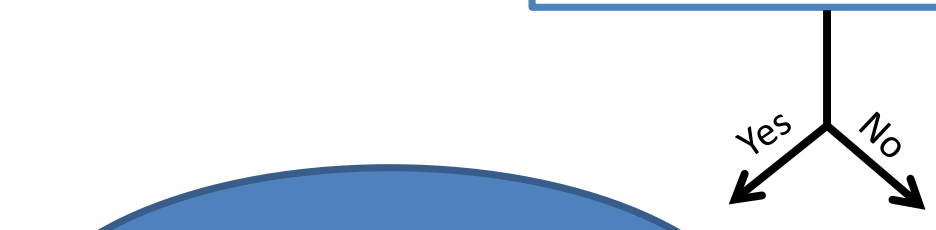


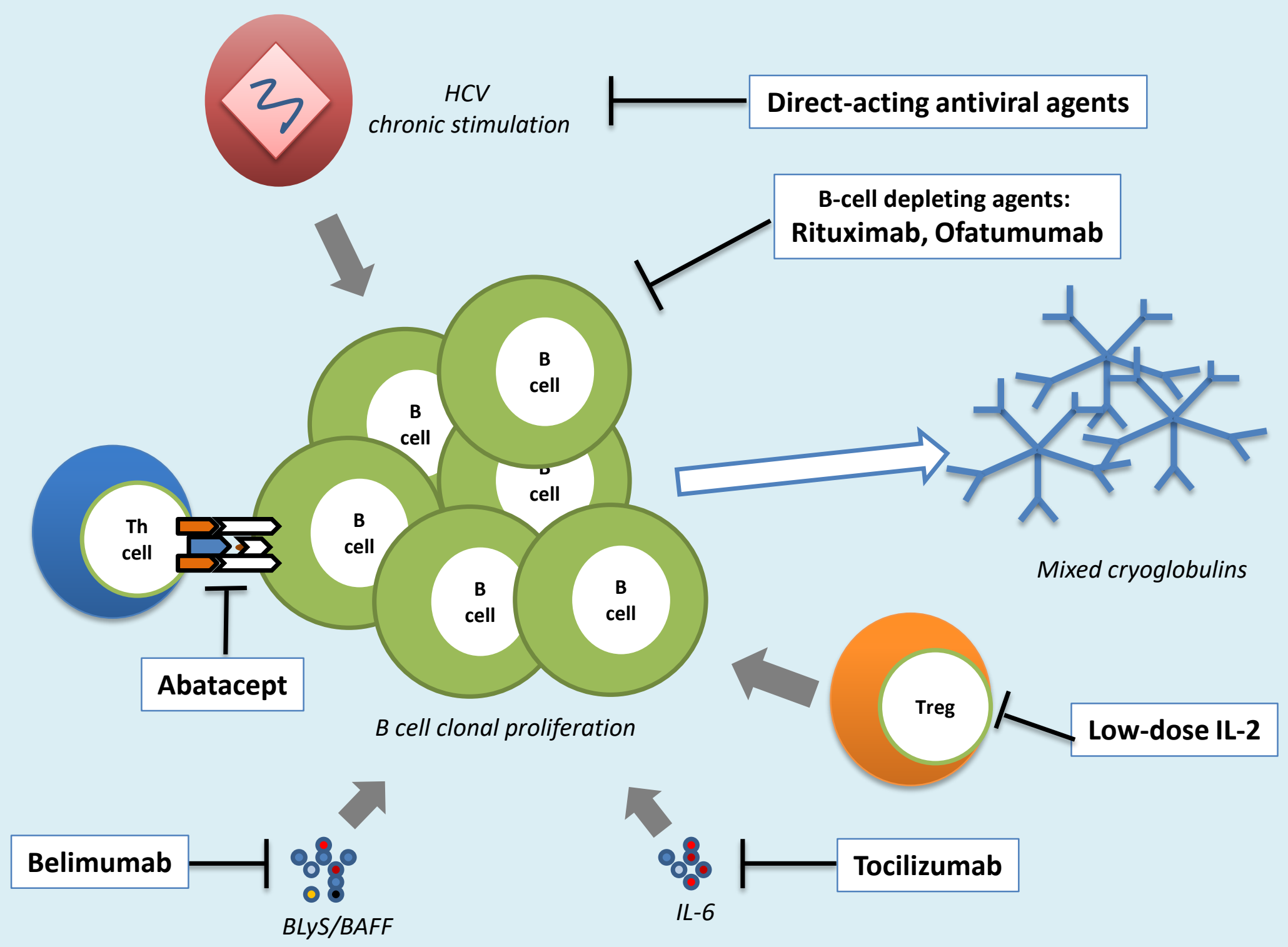

